\title{
ESTRUCTURA DE LA COMUNIDAD DEL PHYLUM ECHINODERMATA EN AGUAS SOMERAS DE LA BAHÍA DE TAGANGA, CARIBE COLOMBIANO
}

\section{SHALLOW WATER ECHINODERMATA PHYLUM COMMUNITY STRUCTURE OF TAGANGA BAY, COLOMBIAN CARIBBEAN}

Juan D. Gaitán-Espitia1

\section{RESUMEN}

La bahía de Taganga soporta una fuerte presión antropogénica que amenaza a las comunidades y ecosistemas marinos establecidos en este sector, especialmente a organismos sensibles a cambios ambientales y contaminación, como los equinodermos. En este estudio, se determinó la estructura de la comunidad del phylum Echinodermata en las aguas someras de la bahía de Taganga, con el fin de establecer una línea base para el monitoreo del impacto de las actividades humanas sobre la fauna marina de este sector turístico. Para el reconocimiento de las principales especies de este Phylum, se realizaron salidas de campo desde enero a septiembre de 2006, identificando los organismos presentes en el área, mediante censo visual y colecta manual. Los muestreos fueron efectuados en la zona intermareal a profundidades menores a $10 \mathrm{~m}$; los 283 especimenes colectados fueron identificados en el laboratorio del Centro de Investigaciones en Zoología y Ecología Marina en la Universidad del Magdalena, siguiendo las claves correspondientes para cada clase, género y especie. Se presenta una lista sistemática que incluye 18 especies de equinodermos distribuidas en cinco clases y doce familias para esta localidad;

${ }^{1}$ Biólogo Marino. Master en Ciencias y Tecnologías Marinas, Estudiante de Doctorado en Sistemática y Ecología de la Universidad Austral de Chile. Director Centro de Investigaciones en Zoología y Ecología Marina (CIZEM). K $3 \mathrm{~N}^{\circ}$ 7-63 Apto 1401, Rodadero - Santa Marta. PBX: + (57) 5 4229975. E-mail: jgaitan@unimagdalena.edu.co. siendo las clases Echinoidea y Ophiuroidea las mejor representadas en términos de riqueza, con siete y seis especies, respectivamente, al igual que de abundancia relativa

Palabras clave: Equinodermos, Caribe colombiano, diversidad, zona intermareal, aguas someras, Taganga.

\section{SUMMARY}

The Taganga bay supports a strong anthropogenic pressure that threatens the communities and marine ecosystems established in this sector, especially organisms sensible to environmental changes and contamination like the echinoderms. In this study, the structure of the Phylum Echinodermata community in shallows waters of Taganga bay was determined, with the purpose of establishing a base line for monitoring the human activities impact on the marine fauna of this tourist sector. For the survey of the main species of this phylum, field trips were organized from January to September of 2006, identifying the existing species of echinoderms in the area by visual census and manual collects. The samplings were taken in the intermareal zone up to a depths of $<10 \mathrm{~m}$; the 283 specimens collected were identified in the laboratory of CIZEM at the Magdalena University following the corresponding keys for each class and genus. A systematic list is presented for this locality that includes 18 species of echinoderms distributed in five classes and twelve, being the Class Echinoidea and Ophiuroidea the best represented in terms of richness with seven and six species respectively, as well as of relative abundance. 
Key words: Echinoderms, Colombian Caribbean, diversity, intermareal zone, shallows waters, Taganga.

\section{INTRODUCCIÓN}

Uno de los grupos taxonómicos de mayor importancia en la estructura de las comunidades marinas es el Phylum Echinodermata. Su presencia es conspicua en todos los ambientes marinos, desde la zona intermareal hasta los abismos oceánicos y desde las fuentes hidrotermales submarinas hasta las aguas polares (Hooker et al. 2005). Aproximadamente existen 16 clases extintas, con más de 13.000 especies fósiles descritas. Al menos 6.500 especies de equinodermos viven aun distribuidas en cinco clases reconocidas. Las estrellas de mar pertenecen a la Clase Asteroidea, con aproximadamente 1.800 especies vivas; los ofiuroideos conforman la Clase Ophiuroidea, con aproximadamente 2.000 especies actuales, la Clase Crinoidea esta compuesta actualmente por aproximadamente 700 especies, mientras que la Clases Echinoidea y Holothuroidea presentan, en la actualidad cerca de 900 y 1.200 especies respectivamente (Hendler et al. 1995).

Los equinodermos son un grupo de organismos que evolutivamente se han adaptado a ambientes costeros, constituyendo un eslabón importante dentro de las redes tróficas y ejerciendo una influencia considerable sobre el ecosistema. Su éxito en la colonización del mar, se debe a las adaptaciones que han venido desarrollando desde su aparición antes del Cámbrico Inferior (Hooker et al. 2005). Todos los equinodermos son marinos $y$, excepcionalmente, viven en las desembocaduras de algunos esteros; la invasión del medio dulceacuícola ha sido restringida por su intercambio gaseoso a nivel epidérmico y por la ausencia de estructuras excretoras y osmoreguladoras. El grupo muestra una amplia variedad de estrategias alimenticias, desde la captación de nutrientes por absorción corporal, alimentadores de depósito y suspensión, herbívoros, detritívoros, prededadores oportunistas, comensalismo, hasta especialistas estrictos, como Solaster stimpsonii, una estrella de mar del Pacífico Noreste, que se alimenta exclusivamente de una especie de pepino de mar (SolísMarín, 1997). El rol de los equinodermos es fundamental para el equilibrio y la dinámica en los ecosistemas marinos, por ejemplo, poblaciones densas de erizos y estrellas de mar pueden devastar trayectos de algas, pastos marinos y corales, favoreciendo la colonización de otros organismos. Adicionalmente, la pesca intensiva de peces asociados a arrecifes de coral, favorece el incremento en las poblaciones de equinodermos y más específicamente de erizos y estrellas de mar, los cuales pueden llegar a afectar seriamente el estado de estos ecosistemas marinos deteniendo la recuperación de las formaciones coralinas. La restauración de estos arrecifes podría ser acelerada interviniendo y reduciendo la abundancia de las poblaciones de erizos marinos, particularmente, de las especies longevas. Si los arrecifes están seriamente degradados o se reduce o elimina el esfuerzo pesquero, se recomienda la reducción en el número de erizos marinos para la restauración del número de peces, la diversidad de especies y la producción pesquera. Sin embargo, más allá de la problemática ecológica que pueden ocasionar el incremento descontrolado en las poblaciones de ciertos equinodermos, hay que entender que los erizos y los pepinos de mar son responsables de la producción de sedimentos en los fondos marinos, contribuyendo a los flujos de energía en la zona bentónica, por lo cual, son organismos fundamentales para el equilibrio de estos ecosistemas (Hendler et al. 1995; McClanahan et al. 1996, Mamelona \& Pelletier, 2005; Gagnon et al. 2003; Edmunds \& Carpenter, 2001).

Los equinodermos representan un creciente potencial económico para la industria pesquera, por lo cual, se han incrementado las actividades orientadas a su explotación. Sin embargo, es poco lo que se conoce sobre la biología y la ecología de estos organismos $\mathrm{y}$, al adicionar la no existencia de regulaciones a nivel mundial ni en Colombia que controlen la explotación y el comercio de pepinos de mar y erizos, se amenaza el estado de las poblaciones, como ocurrió en Galápagos - Ecuador, con Isostichopus fuscus y Tripneustes depressus, por la fuerte presión de la pesquería artesanal (Cruz et al. 2003).

El conocimiento del phylum Echinodermata en el Caribe colombiano ha presentado un considerable incremento en los últimos años, tanto en aguas someras como profundas. Se han realizado trabajos, como el de Allain (1976), para la parte Norte de Colombia, Gallo (1988), para el Parque Nacional Natural Tayrona, al igual que el listado preliminar de equinodermos del Caribe colombiano presentado por Álvarez (1981), el trabajo de González et al. (2002), en la zona de Cartagena y el Golfo de Urabá, la investigación sobre hábitats de oxiuros, por 
Bejarano et al. (2004), y el trabajo de Benavides-Serrato et al. (2005), a lo largo de la plataforma continental y talud superior.

Es difícil definir puntualmente el estado de las poblaciones de equinodermos en aguas colombianas, no obstante, debido a los efectos de las actividades antropogénicas, como la pesca, la urbanización de zonas costeras, los vertimientos de aguas residuales, entre otros, la fauna y flora marina han sufrido un considerable deterioro. Más específicamente, en el caso de los equinodermos la explotación comercial a nivel mundial para consumo humano ha aumentado significativamente en los últimos años (Jenkins \& Mulliken, 1999), mientras que en Colombia no existe una cultura generalizada de consumo. Sin embargo, las estadísticas manejadas por la FAO son desactualizadas y en Colombia, el constante cambio de las autoridades responsables del sector pesquero, no ha permitido la consolidación de una base de datos concreta sobre la captura de estos organismos. Por esta razón es vital el desarrollo de proyectos de investigación enfocados en aspectos biológicos, ecológicos y pesqueros que orienten a los órganos gubernamentales para la toma de decisiones y la gestión de estos recursos naturales, como ocurre en el sector turístico de la Bahía de Taganga, donde la construcción del Colector Pluvial ha comenzado a generar problemas ambientales que pueden ser monitoreados mediante bioindicadores, como los equinodermos.

Este trabajo brinda información taxonómica y ecológica de las especies del phylum Echinodermata presentes en la bahía de Taganga a profundidades menores de $10 \mathrm{~m}$, antes de la implementación del Colector Pluvial de Santa Marta, sirviendo de base a estudios posteriores de monitoreo ambiental y ampliando el conocimiento de la fauna marina de esta región.

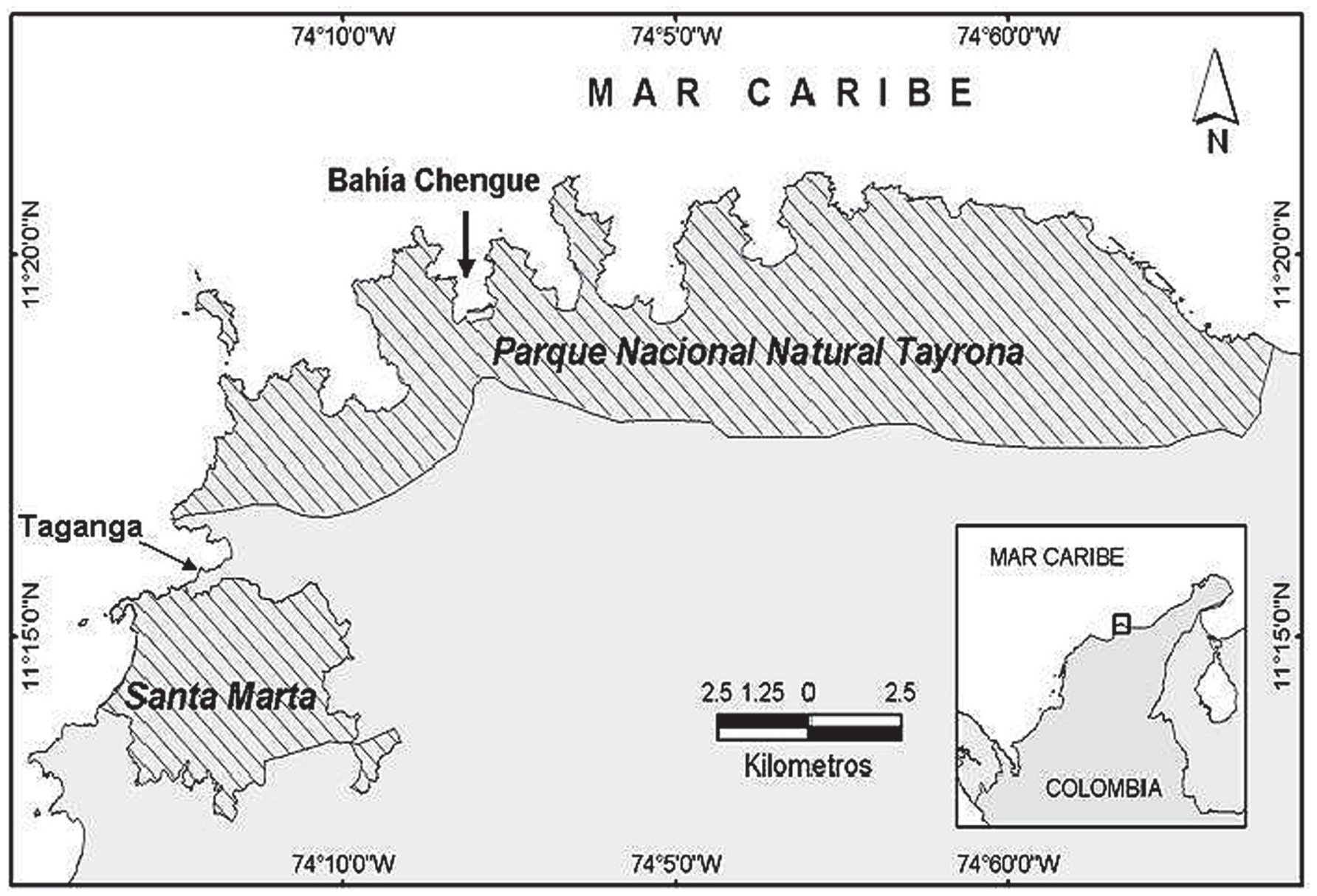

Figura 1. Ecorregión Tayrona (Modificado de Díaz-Pulido \& Garzón-Ferreira, 2002). 


\section{MATERIALES Y MÉTODOS}

Descripción del área: La bahía de Taganga se encuentra ubicada entre los $11^{\circ} 15^{\prime} 43.61^{\prime \prime} \mathrm{N}, 74^{\circ} 11^{\prime} 56.59^{\prime \prime} \mathrm{W}$ y los $11^{\circ} 16$ '14.39”N, 74¹2'18.82” W (Figura 1). Es una bahía formada por estribaciones de la Sierra Nevada de Santa Marta. Presenta una amplia variedad de habitat que van desde asociaciones coralinas con varias especies, entre ellas Porites porites, P. astreoides, Montastrea annularis, Diploria strigosa, D. labyrinthiformis, Meandrina meandrites, entre otros, hasta fondos arenosos, con materia orgánica y sedimentos provenientes de la parte continental y de los procesos naturales del ecosistema marino, propiciando la diversidad de especies y las relaciones bióticas, entre estos biotopos. Esta bahía también recibe una fuerte carga antropogénica por parte del turismo por ser de fácil acceso en comparación con otras ubicadas más al norte de la capital del departamento, por el atractivo visual que brinda a los visitantes y por la pesca artesanal ejercidas por los pobladores de la zona (Agenda Caribe, 2006).

Trabajo de campo: Entre enero y septiembre de 2006, se realizaron diez transectos aleatorios de 50 metros mensualmente, observando especies presentes, en las diferentes ensenadas y accidentes geomorfológicos de la Bahía de Taganga, con el fin de obtener material y datos biológicos de las especies de equinodermos que habitan en la zona intermareal a profundidades menores a $10 \mathrm{~m}$, mediante buceo a pulmón libre, en jornadas diurnas y nocturnas, tomando datos de abundancia y caracterizando relaciones funcionales con otros organismos, como peces, algas, pastos y corales. Los especimenes colectados fueron anestesiados y fijados en alcohol etílico al $70 \%$, preparado con agua destilada. Estos animales fueron identificados en el laboratorio del Centro de Investigaciones en Zoología y Ecología Marina en la Universidad del Magdalena, con las guías correspondientes para cada clase: Hendler et al. (1995) y Caycedo (1979) para ofiuros y holoturidos y Smith (2005), para equinoideos. Las clases están dispuestas de acuerdo a Smith (1984). Los órdenes y las familias se dispusieron teniendo en cuenta los estudios de Serafy (1979) y Hendler et al. (1995). Todos los ejemplares fueron almacenados en la colección de fauna marina del Centro de Investigaciones en Zoología y Ecología Marina, CIZEM.
Trabajo de gabinete: La comunidad de equinodermos, se caracterizó mediante la frecuencia y la abundancia relativa, de donde se derivó el análisis de la diversidad, la uniformidad y la riqueza. Por la necesidad de caracterizar la estructura de la comunidad, se calcularon los índices de diversidad (H'), basados en el concepto de Wilhm (1975), quien expresa que las unidades de diversidad dependen de la base de logaritmos utilizados; para el presente trabajo fue aplicado el logaritmo base dos, expresando la diversidad como contenido de información en bits por individuo, tomando valores de 0 a 5 (Caso-Muñoz et al. 1995). Este índice ha sido comúnmente utilizado en muchos trabajos dirigidos hacia el estudio de las comunidades bénticas (Pielou, 1975; Gray, 1981; CasoMuñoz et al. 1995 y Thiel $E$ Ullrich, 2002). Igualmente, se utilizó el índice de Margalef (DMg), uniformidad (E), riqueza de especies (S), mediante la aplicación de los índices y valores propuestos por Shannon-Wiener (1963), Brower \& Zar (1977) y Margalef (1958), respectivamente. Para la dominancia, se utilizaron los índices de Simpson y Berger-Parker (Juncos et al. 2006).

\section{RESULTADOS Y DISCUSIÓN}

Según Moreno (2001), la riqueza específica (S) es la forma más sencilla de medir la biodiversidad, ya que se basa únicamente en el número de especies presentes, sin tomar en cuenta el valor de importancia de las mismas, hallándose en el presente estudio 18 especies, con un total de 283 ejemplares, distribuidos en cinco clases, nueve órdenes y doce familias (Tabla 1).

De la equinofauna registrada para aguas someras $(<10 \mathrm{~m})$ en el sector de la bahía de Taganga, la mayor riqueza fue registrada para la Clase Echinoidea con siete especies, seguida por la Clase Ophiuroidea (Figura 2). Por otro lado, la Clase Holothuroidea representó cerca del 7,4\% $(n=21)$ del total de ejemplares colectados (Tabla 1) y ocupó el tercer lugar en representatividad de especies de la equinofauna registrada (Figura 2). Los crinoideos y asteroideos presentaron las menores riquezas con una sola especie, registrada para cada clase en abundancias del 0,7 y 6\%, respectivamente, con los organismos Nemaster rubiginosus y Linckia guildingii (Tabla 1). La abundancia más alta fue registrada en la Clase Echinoidea con 152 organismos, siendo la especie Lytechinus variegatus, la más numerosa (Tabla 1). Con relación a otros indicadores ecológicos, se encontró que el índice de Simpson (DSp) presentó un valor de 
Tabla 1. Composición y abundancia del Phylum Echinodermata de aguas someras de la bahía de Taganga ( $\mathrm{N}=$ Número de organismos, $\% \mathrm{~A}=\%$ abundancia relativa).

\begin{tabular}{|c|c|c|c|c|}
\hline CLASE & $\begin{array}{l}\text { ORDEN } \\
\text { FAMILIA }\end{array}$ & ESPECIE & $\mathbf{N}$ & $\% \mathrm{~A}$ \\
\hline $\begin{array}{c}\text { Crinoidea Miller, } \\
1821\end{array}$ & $\begin{array}{c}\text { Comatulida A. H. Clark, } 1908 \\
\text { Comasteridae Clark, } 1908\end{array}$ & Nemaster rubiginosus (Pourtalès, 1869) & 2 & 0,7 \\
\hline $\begin{array}{l}\text { Asteroidea de } \\
\text { Blainville, } 1830\end{array}$ & $\begin{array}{c}\text { Valvatida Perrier, } 1884 \\
\text { Ophidiasteridae Verrill, } 1867\end{array}$ & Linckia guildingii Gray, 1840 & 17 & 6 \\
\hline \multirow{6}{*}{$\begin{array}{l}\text { Ophiuroidea } \\
\text { Gray, } 1840\end{array}$} & $\begin{array}{l}\text { Ophiurida Müller and Troschel, } 1840 \\
\text { Ophiocomidae Ljungman, } 1867\end{array}$ & Ophiocoma echinata Lamarck, 1816 & 31 & 10,9 \\
\hline & & O. pumila Lütken, 1859 & 6 & 21 \\
\hline & & O. wendtii Müller y Troschel, 1842 & 12 & 4,2 \\
\hline & Ophionereididae Ljungman, 1867 & Ophionereis reticulata (Say, 1825) & 29 & 10,2 \\
\hline & \multirow{2}{*}{ Ophiodermatidae Ljungman, 1867} & Ophioderma apressum (Say, 1825) & 5 & 1,7 \\
\hline & & O. cinereum Müller y Troschel, 1842 & 8 & 2,8 \\
\hline \multirow{6}{*}{$\begin{array}{l}\text { Echinoidea } \\
\text { Leske, } 1778\end{array}$} & $\begin{array}{r}\text { Cidaroida Claus, } 1889 \\
\text { Cidaridae, Gray } 1825\end{array}$ & Eucidaris tribuloides Lamarck, 1816 & 23 & 8,1 \\
\hline & \multirow{2}{*}{$\begin{array}{l}\text { Diadematoida Duncan, } 1889 \\
\text { Diadematidae Gray, } 1855\end{array}$} & Diadema antillarum Philippi, 1845 & 37 & 13 \\
\hline & & Astropyga magnifica Clark, 1934 & 4 & 1,4 \\
\hline & $\begin{array}{c}\text { Temnopleuroida Mortensen, } 1942 \\
\text { Toxopneustidae Troschel, } 1872\end{array}$ & $\begin{array}{l}\text { Lytechinus variegatus Lamarck, } 1816 \\
\text { Tripneustes ventricosus Lamarck, } 1816\end{array}$ & $\begin{array}{l}43 \\
21\end{array}$ & $\begin{array}{c}15,2 \\
7,4\end{array}$ \\
\hline & \multirow{2}{*}{$\begin{array}{l}\text { Echinoida Claus, } 1876 \\
\text { Echinometridae Gray, } 1825\end{array}$} & Echinometra lucunter Linnaeus, 1758 & 16 & 5,6 \\
\hline & & E viridis Agassiz, 1863 & 8 & 2,8 \\
\hline \multirow[t]{2}{*}{$\begin{array}{l}\text { Holothuroidea de } \\
\text { Blainville, } 1834\end{array}$} & $\begin{array}{c}\text { Aspidochirotida Grube, } 1840 \\
\text { Stichopodidae Haeckel, } 1896 \\
\text { Holothuriidae Ludwig, } 1894\end{array}$ & $\begin{array}{l}\text { Isostichopus badionotus Selenka, } 1867 \\
\text { Holothuria thomasi Pawson y Caycedo, } 1980\end{array}$ & $\begin{array}{c}13 \\
6\end{array}$ & $\begin{array}{l}4,6 \\
2,1\end{array}$ \\
\hline & \multirow[t]{2}{*}{$\begin{array}{r}\text { Dendrochirotida Grube, } 1840 \\
\text { Cucumariidae Ludwig, } 1894\end{array}$} & Leptopentacta deichmannae Domantay, 1953 & 2 & 0,7 \\
\hline & & & 283 & 100 \\
\hline
\end{tabular}

0,0853, lo cual al considerar que es un índice que toma en cuenta la representatividad de las especies con mayor valor de importancia, es decir, que está influido por la importancia de las especies más dominantes, indicaría que la equinofauna de las aguas someras de la bahía de Taganga, no presenta una dominancia ecológicamente alta, lo cual se corrobora con el índice de uniformidad (E) hallado para el área de estudio que fue de 0,8989 y el índice de Shannon $\left(\mathrm{H}^{\prime}\right)$ que fue de 2,5983, asumiendo que este último adquiere valores entre cero, cuando hay una sola especie y el logaritmo de $S$, cuando todas las especies están representadas (Moreno, 2001), lo cual, en el presente estudio equivaldría a 2,89 , siendo un valor muy cercano al encontrado. Otros índices utilizados, como el Alfa (distribución logarítmica) y el índice de Margalef, señalan valores relativamente altos de riqueza, corroborándose con valores bajos en el índice de dominancia de Berger-Parker (Tabla 2).

La composición faunística de los equinodermos en la Bahía de Taganga presenta similitudes con otras de la misma naturaleza, como el Parque Nacional Natural 


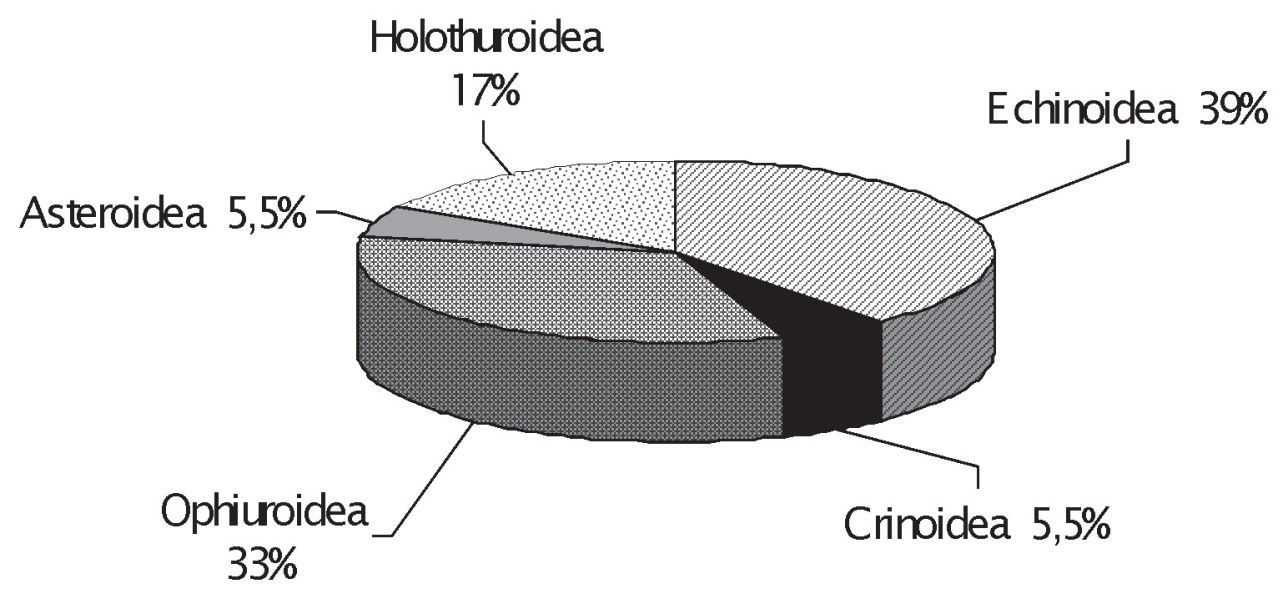

Figura 2. Representación porcentual de la riqueza de las distintas clases de equinodermos recolectados en la zona intermareal de la bahía de Taganga a profundidades menores de $10 \mathrm{~m}$.

Tabla 2. Índices ecológicos de diversidad para la equinofauna presente en aguas someras de la bahía de Taganga.

\begin{tabular}{|l|c|}
\hline \multicolumn{1}{|c|}{ Índices Ecológicos } & Valor \\
\hline Número de individuos & 283 \\
\hline Riqueza de especies (S) & 18 \\
\hline Uniformidad ( E ) & 0,8989 \\
\hline Índice de Margalef (DMg) & 3,0112 \\
\hline Índice de Simpson (DSp) & 0,0853 \\
\hline Inverso de Simpson (1/DSp) & 11,1721 \\
\hline Índice de Shannon (H') & 2,5983 \\
\hline Varianza de Shannon & 0,0015 \\
\hline Índice de Berger-Parker (d) & 0,1519 \\
\hline Inverso de Berger-Parker (1/d) & 6,5814 \\
\hline Alfa (Distribución logarítmica) & 4,2783 \\
\hline
\end{tabular}

Tayrona y otras ensenadas del Caribe colombiano (Borrero-Pérez et al. 2002a, 2002b; Caycedo, 1978; 1979) y concuerda con estudios hechos en todo el caribe (Hendler et al. 1995). Los equinoideos y los ofiuroideos son organismos que se distribuyen ampliamente a lo largo del litoral rocoso, sobre corales, debajo de piedras y de guijarros, por lo cual, su captura es sencilla y permite obtener gran número de ejemplares y especimenes, como se evidenció en el presente trabajo, al ser las clases con mayores índices de riqueza y abundancia, 
comparado con otros equinodermos, como los crinoideos y asteroideos.

La distribución de los equinodermos en diversos gradientes ambientales y hábitat, se relaciona con el tipo de alimentación que presentan dichos organismos (Caso-Muñoz et al. 1995). De las especies que conforman la equinofauna de aguas someras de la bahía de Taganga, un elevado porcentaje (72\%), corresponde a organismos infaunales y seminfaunales, como son aquellos pertenecientes a las Clases Echinoidea y Ofiuroidea, mientras que tres clases restantes corresponden a formas epifaunales y representan el $28 \%$ de la equinofauna de la zona.

Algunas especies de erizos encontradas, como $E$. tribuloides, D. antillarum, L. variegatus, T. ventricosus, $E$. lucunter, E. viridis, se caracterizan por vivir en pequeñas grietas de los arrecifes de coral y de formaciones rocosas, en zonas de pastos marinos y se alimentan de algas, de corales, de briozoos y de fanerógamas marinas (Hendler et al. 1995). La presencia de algunas especies, como $D$. antillarum en ecosistemas marinos, es vital debido a que al ser netamente herbívoro, juega un papel ecológico muy importante regulando el crecimiento de algas y facilitar el asentamiento de larvas de coral (Lessios et al. 2001).

Tripneustes ventricosus presentó altas densidades durante toda la época de muestreo (tanto en el día como en la noche), se distribuyó a lo largo del gradiente de profundidad y se pudo observar una fuerte asociación con $L$. variegatus, que al igual que Echinometra lucunter, soportan fuerte oleaje y pueden encontrarse a poca profundidad. E. lucunter, se caracteriza por ser excavador, lo cual lo convierte en un factor significante en la bioerosión de los arrecifes de coral. Esta especie es potencialmente útil como bioindicador, ya que algunos individuos han mostrado que han acumulado cierta cantidad de metales pesados en sus gónadas, caparazón, espinas y linterna de Aristóteles, reflejando el grado de exposición a contaminantes ambientales, como $\mathrm{Pb}$ y $\mathrm{Cd}$ que son tóxicos y peligrosos para la salud humana al ser ingeridos (González et al. 1999).

El pepino de mar Holothuria thomasi y la estrella Linckia guildingii evidenciaron tener una fuerte asociación con formaciones coralinas y rocosas, donde permanecen inactivos la mayor parte del día, entre grietas, probablemente como estrategia de protección, mientras que el holoturido Isostichopus badionotus solo se encontró asociado a zonas arenosas. En los tres casos fue muy claro que las densidades aumentan a medida que aumenta la profundidad, mostrando mayor actividad en la noche, probablemente esto se asocie a su etología y ecología alimenticia, porque en el caso de Linckia guildingii, se alimenta de pólipos de coral, los cuales salen de sus estructuras calcáreas en la noche y tanto Holothuria thomasi como Isostichopus badionotus son organismos epibentónicos sedimentívoros (Sambrano et al. 1995), que aprovechan las concentraciones de fanerógamas marinas en fondos arenosos y lodosos para alimentarse.

Todos los especimenes encontrados de la Clase Ophiuroidea, se caracterizaron por permanecer durante el día debajo de las rocas en grupos muy numerosos y en el transcurso de la noche, se desplazaron sobre el coral de manera activa. Se observó que son presas principalmente de peces lutjanidos, sparidos, carangidos y de cangrejos. En el caso de Nemaster rubiginosus, solamente pudo ser encontrado en muestreos nocturnos, cuando permanecía fuera de las cavidades rocosas, alimentándose de plancton y partículas en suspensión.

Conociendo la composición e importancia de la macrofauna de los ecosistemas marinos presentes en la región de Santa Marta y más específicamente en la bahía de Taganga es necesario desarrollar otros trabajos enfocados a la ecología de los equinodermos, teniendo en cuenta el tipo de fondo, variaciones climáticas, temperatura, entre otros factores, que permitan tener una mayor dimensión del estado actual de sus poblaciones, comparado con áreas protegidas aledañas, como el Parque Nacional Natural Tayrona.

\section{AGRADECIMIENTOS}

Al estudiante de Biología Hugo Llanos de la Universidad del Magdalena, al igual que a todos los integrantes del Centro de Investigaciones en Zoología y Ecología Marina (CIZEM), por el acompañamiento en campo y laboratorio. 


\section{BIBLIOGRAFÍA}

AGENDA CARIBE. 2006. Grave amenaza a la vida y al medio ambiente en Taganga, Magdalena. Disponible desde Internet en http://agendacaribe. blogspot.com/2006_09_01_archive.html (con acceso el 13/08/07)

ALLAIN, J. 1976. Erizos de la Costa Norte de Colombia. Informe Museo del Mar. 15:1-18.

ÁLVAREZ, L.R. 1981. Listado preliminar de los equinodermos de la costa Atlántica colombiana. Bol. Museo del Mar. 10:24-39.

BEJARANO, S.; ZEA, S.; DÍAZ, J.M. 2004. Esponjas y otros microhábitats de ofiuros (Ophiuroidea: Echinodermata) en ambientes arrecifales del Archipiélago de San Bernardo (Caribe colombiano). Bol. Invest. Mar. Cost. 33:29-47.

BENAVIDES-SERRATO, M.; BORRERO-PÉREZ, G.; SOLANO, O.; NAVAS, G. 2005. Listado taxonómico de los asteroideos (Echinodermata: Asteroidea) de la plataforma y el talud superior del Caribe colombiano. Rev. Biol. Trop. 53(3):171194.

BORRERO-PÉREZ, G.; SOLANO, O.; BENAVIDESSERRATO, M. 2002a. Lista revisada de los erizos (Echinodermata: Echinoidea) del mar caribe colombiano. Biota Colombiana. 3(1):141-148.

BORRERO-PÉREZ, G.; BENAVIDES-SERRATO, M.; SOLANO, O.; NAVAS, G. 2002b. Equinoideos (Echinodermata: Echinoidea) colectados en la franja superior del talud continental del caribe colombiano. Bol. Inv. Mar. Cos. 31:133-166.

BROWER, J.; ZAR, J.. 1977. Field and laboratory methods for general ecology. Brown Comp. 185p.

CASO-MUÑOZ, M.; LAGUARDA, A.; SOLÍS, F.; ORTEGA, A.; DURÁN, A. 1995. Contribución al conocimiento de la ecología de las comunidades de equinodermos de la bahía de Mazatlán, Sinaloa, México. Ann. Inst. Cienc. Mar y Limnol. (México). 22(1):101-119
CAYCEDO, I. E. 1978. Holoturoidea (Echinodermata) de aguas someras en la costa norte de Colombia. An. Ins. Inv. Mar. Punta Betín. 10:149-198.

CAYCEDO, I.E. 1979. Observaciones de los equinodermos en las Islas del Rosario. An. Ins. Inv .Mar. Punta Betín. 11:39-47.

CRUZ, M.; GABOR, N.; MORA, E.; JIMÉNEZ, R.; MAIR, J. 2003. The known and unknown about marine biodiversity in Ecuador (continental and insular). Gayana. 67(2):232-260.

DÍAZ-PULIDO, G.; GARZÓN-FERREIRO,J. 2002. Seasonality in algal assemblages on upwelling-influenced coral reefs in the Colombian Carabbean. Botánica Marina. 45:284-292.

EDMUNDS, P.; CARPENTER, R. 2001. Recovery of Diadema antillarum reduces macroalgal cover and increases abundance of juvenile corals on a Caribbean reef. Proc. Nal. Acad. Sci. 98:5067-5071.

GAGNON, P.; HIMMELMAN, J.; JONSON, L. 2003. Algal colonization in urchin barrens: defense by association during recruitment of the brown alga Agarum cribrosum. J. Exp. Marine Biol. Ecol. 209:179-196.

GALLO, J. 1988. Contribución al conocimiento de los equinodermos del Parque Nacional Natural Tayrona: I Echinoidea. Trianea.1:99-118.

GONZÁLEZ, H.; POMARES, M.; RAMÍREZ, M.; TORRES, I. 1999. Heavy metals in organisms and sediments from the discharge zone of the submarine sewage outfall of Havana City, Cuba. Marine Pollution Bull. 38(11):1048-1051.

GONZÁLEZ, D.; SOLANO, O.D.; NAVAS, G. 2002. Equinodermos colectados por la expedición $\mathrm{CIOH}$ INVEMAR- SMITHSONIAN entre Cartagena y el Golfo de Urabá (29-380m), Caribe colombiano. Bol. Investig. Marinas y Costeras 31:85-132.

GRAY, J. S. 1981. The Ecology of marine sediments. An introduction to the structure and function of benthic communities. Cambridge Univ. Press. 185pp. 
HENDLER, G.; MILLER, J.; PAWSON, D.; KIER, P. 1995. Sea stars, sea urchins and allies. Echinoderms of Florida and the Caribbean. Smithsonian Institution. Washington. 195p.

HOOKER, Y.; SOLIS-MARIN, F.; LLEELLIS, M. 2005. Equinodermos de las Islas Lobos de Afuera (Lambayeque, Perú). Rev. Peru. Biol. 12(1):77-82

JENKINS, M.; MULLIKEN, T. 1999. La Evolución de la explotación en las Islas Galápagos: El Comercio del Pepino de Mar de Ecuador. Traffic Bulletin, 17(3):107-118.

JUNCOS, R.; BISTIONI, M.; FREYER, I. 2006. Composición y estructura de la ictiofauna del Río CeballosSaldán (Córdoba, Argentina). Iheringia, Sér. Zool., Porto Alegre. 96(3):363-371.

LESSIOS, H.A.; KESSING, B.D.; PEARSE, J.S. 2001. Population structure and speciation in tropical seas: Global phylogeography of the sea urchin Diadema. Evolution. 55(5):955-975.

MAMELONA, J.; PELLETIER, E. 2005. Green urchin as a significant source of fecal particulate organic matter within nearshore benthic ecosystems. J. Exp. Marine Biol. Ecol. 314:163-174.

MARGALEF, R. 1958. General Systematics. Information theory in ecology. 3:36-71.

MCCLANAHAN, R.; KAMUKURU, A.; MUTHIGA, N.; GILAGABHER, M.; OBURA, D. 1996. Effect of Sea Urchin Reductions on Algae, Coral, and Fish Populations Conservation Biology 10(1):136-154.

MORENO, C. 2001. Métodos para medir la biodiversidad. MET Manuales y tesis SEA Vol. 1. Zaragoza, 84 p.

PIELOU, E.C. 1975. Ecological Diversity. John Wiley \& Sons, Inc., New York, 165p.
SAMBRANO, A.; DÍAZ, H.; CONDE, J.E. 1995. Caracterización de la ingesta en Isostichopus badionotus (Salenka) y Holothuria mexicana Ludwig (Echinodermata: Holothuroidea). Caribbean J. Sci. 26(1-2):45-51.

SERAFY, K.D. 1979. Echinoids (Echinodermata: Echinoidea). Memoirs of the Hourglass Cruises. 5:1-120.

SHANNON, E.; WIENER, N. 1963. The mathematical theory of communication. University of Illinois Press Urbana. 117p.

SOLÍS-MARÍN, F.A. 1997. Catálogo de los equinodermos recientes de México. Informe Técnico del Proyecto CONABIO, Instituto de Ciencias del Mar y Limnología, Universidad Autónoma de México. 26p.

SMITH, A.B. 1984. Classification of the Echinodermata. Paleontology, 27:431-459

SMITH, A.B. 2005. The Echinoid Directory. World Wide Web electronic publication.Disponible desde Internet en http://www.nhm.ac.uk/research-curation/projects/echinoid-directory/intro/introduction. html (con acceso 13/11/06)

THIEL, M.; ULLRICH, N. 2002. Hard rock versus soft bottom: The fauna associated with intertidal mussel beds on hard bottoms along the coast of Chile, and considerations on the functional role of mussel beds. Helgoland Marine Res. 56:21-30

WILHM, J. 1975. River Ecology. Biological indicators of pollution. p.375-402.

Recibido: Septiembre 18 de 2007

Aceptado: Abril 2 de 2008 